\title{
Computerized Neuropsychological Assessments: Pros and Cons
}

\section{To the Editor:}

January 12, 2009

We read with interest the "Computerized Neuropsychological Assessments" article by Woo. ${ }^{1}$ This article highlighted a number of advantages and disadvantages of computerized assessment, including simplicity; precision of measurement; shorter assessment time; standardization; automatic scoring; and minimization of subjectivity, as well the impact examiners may have on participant (advantages); use of multiplechoice formats; reliance on the visual modality; provision of less qualitative information than pencil-and-paper tests; limited assessment of each cognitive domain (ie, one measure to assess a particular cognitive domain); and participant unfamiliarity with computers (disadvantages).

We were surprised by the failure to note crucial sources of error in computerized neuropsychological assessment, ${ }^{2}$ including use of various configurations and operating systems, and real-time versus store-and-forward Internetbased assessment, which highlights the need for standardization of technologies and methods in which Web-based information may be exchanged. We were also surprised by some of the disadvantages raised in this review, ${ }^{1}$ many of which have already been overcome. Modern computerized neuropsychological batteries have already incorporated auditory as well as visual modalities with the simultaneous use of both, have been programmed to provide detailed qualitative information for multiple measures of function within single domains, and have enabled testing on computerized touch screens that are easier to learn and more intuitive.

In addition, we would like to note that multiple-choice testing is not necessarily typical in neuropsychological testing. Computerized neuropsychological testing can employ similar neuropsychological tasks to those employed in paper-and-pencil testing (including, for example, trail-making tests, the cognitive Stroop task, choice reaction time, California Verbal Learning Test, tests of visual and working memory, Controlled Oral Word Association Task, spot the real word test, go/no-go task), which is facilitated by use of a touch screen interfacing with the computer.

Recognition of the value of computerized neuropsychological testing led the American Psychological Association to highlight the six major benefits of computerized assessments, ${ }^{3,4}$ which include automated data collection and storage; greater efficiency of use; release of the clinician from task administration to focus on treatment; greater sense of mastery and control for the client; reduced negative self-evaluation among clients that experience difficulty on the computer; and greater ability to measure aspects of performance not possible through traditional means, such as latency, strength, and variability in response patterns. Computerization provides the unique opportunity for immediate clinical decision support and has been found to significantly improve clinical practice. ${ }^{5}$ Notwithstanding the aforementioned benefits, as noted by Woo, ${ }^{1}$ and the importance of a clinician in the interpretation of the testing output is of clinical imperative.

In this regard, we would like to introduce two other options for computerized assessment not mentioned in Woo's article ${ }^{1}$ : IntegNeuro ${ }^{6}$ and WebNeuro, ${ }^{7}$ which provide automated assessment using standardized specialized hardware and software and Internet-enabled assessment software, respectively. These tools are being used in healthy controls to examine the relationships between gene, brain, and behavior ${ }^{8}$ as well as in a number of clinical populations. ${ }^{9,10}$ Reliability, validity, and norms for comparison with clinical groups for both IntegNeuro $0^{6,11,12}$ and WebNeuro $7,13,14$ have been previously reported. Explicit benefits include standardization, which leads to enhanced reliability and, therefore, accuracy (eg, increased precision of reaction times); 
efficiency (ie, 60 minutes for IntegNeuro and 30 minutes for WebNeuro makes cognitive assessment doable in real-world clinical settings); integration of neuropsychological function with the Brain Resource International Database, ${ }^{15}$ which contains detailed genomic, brain, and behavioral information; and a crucially detailed clinical interpretation that complements the computerized approach.

\section{Sincerely,}

Andrew H. Kemp, PhD

Ainslie Hatch, BA (Hons)

Leanne M. Williams, PhD

\section{REFERENCES}

1. Woo E. Computerized neuropsychalogical assessments. CNS Spectr. 2008;13(10 suppl 16):14-17.

2. Cernich A, Brennana D, Barker L. Bleiberg J. Sources of error in computerized neuropsychological assessment. Arch Clin Neuropsychology. 2007;22:39-48

3. Guidelines for Computer-Based Tests and Interpretation. Washington, DC: American Psychological Association; 1987.

4. American Educational Research Association, American Psychological Association, and the National Council on Measurement in Education. Standards for Educational and Psychological Testing. Washington, DC: American Educational Research Association; 1999.

5. Kawamoto $K$, Houlihan CA, Balas EA, Lobach DF. Improving clinical practice using clinical decision support systems; a systematic review of trials to identify features critical to success. BMJ. 2005;330:765.

6. Paul R, Lawrence J, Williams L, Richard C, Cooper N, Gordon E. Preliminary validity of "integneuro": a new computerized battery of neurocognitive tests. Int $J$ Neurosci. 2005;115:1549-1567

7. Silverstein SM, Berten $S$, Olson $P$, et al. Development and validation of a World-WideWeb-based neurocognitive assessment battery: WebNeuro. Behav Res Methods 2007;39:940-949

8. Gatt JM, Nemeroff CB, Dobson-Stone C, et al. Interactions between BDNF Val66Met polymorphism and early life stress predict brain and arousal pathways to syndromal depression and anxiety. Mol Psychiatry. 2009 Jan 20. [Epub ahead of print].

9. Kemp AH, Gordon E, Rush AJ. Williams LM. Improving the prediction of treatment response in depression: integration of clinical, cognitive, psychophysiological, neuroimaging, and genetic measures. CNS Spectr. 2008:13:1066-1086.

10. Williams LM, Whitford TJ. Flynn G, et al. General and social cognition in first episode schizophrenia: identification of separable factors and prediction of functional outcome using the IntegNeuro test battery. Schizophr Res. 2008;99:182-191.

11. Clark CR, Paul RH, Wiltiams $L M$, et al. Standardized assessment of cognitive functioning during development and aging using an automated touchscreen battery. Arch Clin Neuropsychol. 2006;21:449-467.

12. Williams L, Simms E, Clark C, Paul R, Rowe D, Gordon E. The test-retest reliability of a standardized neurocognitive and neurophysiological test battery: "neuromarker". Int . Nourosci. 2005;115:1605-1630.

13. Mathersul D, Palmer D, Gur $R$, et al. Explicit identification and implicit recognition of facial emotions: Il. Core domains and relationships with general cognition. J Clin Exp Neuropsychol. 2008 Aug 19:1-14. [Epub ahead of print].

14. Williams L, Mathersul D, Palmer D, Gur R, Gur R, Gordon E. Explicit identification and implicit recognition of facial emotions: I. Age effects in males and females across 10 decades. J Clin Exp Neuropsychol. 2008 Aug 19:1-21. [Epub ahead of print].

15. BRAINnet - Brian Research And Integrative Neuroscience Network home page. Available at: http://www. brainnet.net. Accessed January 13, 2009

Dr. Kemp is an NHMRC postdoctoral fellow and head of Depression Research at the Brain Dynamics Centre in the Discipline of Psychological Medicine and Westmead Millennium Institute at the University of Sydney in Australia. Ms. Hatch is a psychologist and is currently completing a PhD at the Brain Dynamics Centre. Dr. Williams is director of the Brain Dynamics Centre.

Disclosures: This research was supported by a National Health and Medical Research Council Project Grant (464863) and an
Australian Research Council Discovery Project (DP0987332). Dr. Kemp is supported by an NHMRC Peter Doherty Fellowship (358770). Dr. Williams is supported by a Pfizer Senior Research Fellowship. Drs. Kemp and Williams have received fees from Brain Resource for work not relating to this publication and hold stock options in Brain Resource. Ms. Hatch reports no financial, academic, or other interest in any organization that may pose a conflict of interest. Brain Resource had no role in the implementation of the publication.

\section{THE AUTHOR RESPONDS}

Kemp and colleagues commented on the "Computerized Neuropsychological Assessments" article, which was based on a discussion from the Alzheimer's Disease Summit. ${ }^{1}$ The purpose of the presentation was to review computer-based measures that have been utilized in the mild cognitive impairment and Alzheimer's disease research literature. Some common advantages and disadvantages of these methodologies were discussed.

They remarked that an additional disadvantage of these assessments includes sources of error from the use of different types of computer software and technologies. It is agreed and already widely accepted that inherent in the use of computers is the potential for multiple pitfalls, such as possible hardware and software failures and lack of equivalence across differing methods. In fact, one central theme of the original presentation was to highlight the important issue that needs to be considered by clinicians and researchers, that traditional paperand-pencil neuropsychological tests may not be analogous to their computer-based counterparts.

Kemp and colleagues also commented that new computer batteries incorporate the simultaneous use of visual and auditory modalities, which does not contradict points from the original article. These points were that the use of a computer entails reliance upon the visual modality, even if auditory presentations are included. In contrast, numerous paper-and-pencil tests are solely auditory, such as the California Verbal Learning Test and the Logical Memory subtest of the Wechsler Memory Scale. If examinees learn a list of words by viewing them on a computer screen and hearing them read aloud, this multimodal type of acquisition may not produce the same type of recall rates as examinees learning the words only by hearing them.

Kemp and colleagues added that computerized assessments have been programmed to assess qualitative aspects of an evaluation. The original article noted that computer tests do not provide as much qualitative information as paper-and-pencil 
testing. While some computerized tests of cognitive ability have incorporated quantitative methods to highlight process aspects of performance, these are limited in contrast those made during a typical neuropsychological assessment. Other researchers ${ }^{2}$ have highlighted the fact that computer tests do not allow the examiner to interrupt the evaluation process, which reduces the examiner's flexibility and ability to "test the limits." Qualitative observations of test performances can be exceedingly helpful in determining the reason for a low test score, in addition to aiding in the identification of an underlying pathological process. Nevertheless, the advantage to the reduced flexibility in computerized assessment is the increased standardization that was discussed in the original article.

Finally, Kemp and colleagues mentioned that multiple-choice testing is not employed in all computerized tests, which is accurate. The main point of the original article was that examinees are often limited to choosing from available response options provided on a computer screen, whether a keyboard or touch screen are the response formats. In paper-and-pencil tests, examinees often have the opportunity to provide spontaneous verbal answers. These answers can include intrusion and perseveration errors that would not be as easily obtained during computerized testing.

Overall, there are advantages and disadvantages to using only paper-and-pencil tests or only computer tests at this time. However, each method provides important information regarding individuals' cognitive functioning and is useful in distinguishing among healthy aging, mild cognitive impairment, and Alzheimer's disease. With advances in technology, computer assessments will likely become even more beneficial than they are currently.

Sincerely, Ellen Woo, PhD

\section{REFERENCES}

1. Woo E. Computerized neuropsychological assessments. CNS Spectr. 2008:13/10 suppl 16):14-17.

2. Schatz P, Browndyke J. Applications of computer-based neuropsychological assessment. J Head Trauma Rehabil. 2002;17:395-410.

Dr. Woo is visiting assistant professor at the Mary S. Easton Center for Alzheimer's Disease Research at the University of California, Los Angeles.

Disclosures: Dr. Woo reports no financial, academic, or other interest in any organization that may pose a conflict of interest. 


\section{Master Psychopharmacology Program}

\section{Puzzle 102}

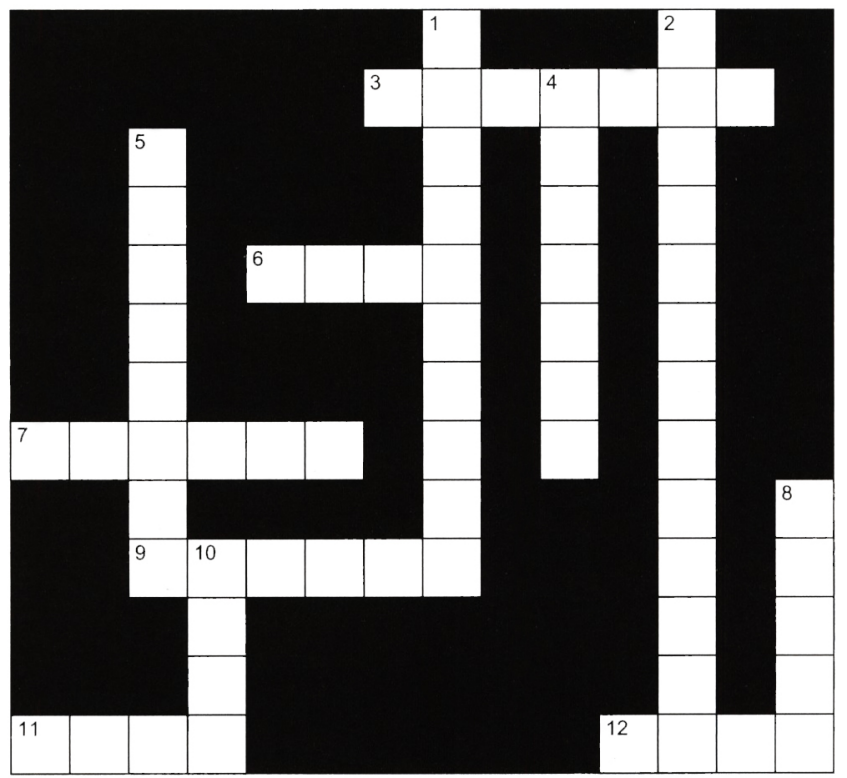

\section{Down}

1. Mu-opioid antagonist that may mitigate euphoria associated with heavy drinking

2. Functionality of dopamine D2 presynaptic receptors

4. Antipsychotics can cause resistance of this hormone

5.

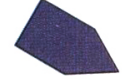

8. Bipolar I disorder requires at least one or mixed episode

10. (abbr.)
Across

3.

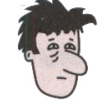

6. Number of subunits that form the alpha pore in a VSSC (voltage-sensitive sodium channel)

7. Bare nerve ending activated only by noxious stimuli

9.

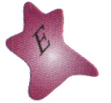

11. Site on neuron that integrates chemical encoding

12. (abbr.)

Think you know?

visit

www.neiglobal.com/go/answers

for answers.

\section{Neuroscience Education Institute}

